(c) 2018 - ISSN 1807-2577

\title{
Avaliação da solubilidade e sorção em água de alguns materiais restauradores diretos
}

\author{
Evaluation of solubility and sorption in water of some direct restorative \\ materials
}

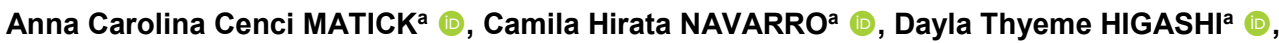 \\ Renata Corrêa PASCOTTO ${ }^{a}$ (D), Mitsue FUJIMAKI ${ }^{a}$ (i), Raquel Sano Suga TERADA* ${ }^{\text {a* }}$ \\ aUEM - Universidade Estadual de Maringá, Maringá, PR, Brasil
}

\begin{abstract}
Como citar: Matick ACC, Navarro CH, Higashi DT, Pascotto RC, Fujimaki M, Terada RSS. Avaliação da solubilidade e sorção em água de alguns materiais restauradores diretos. Rev Odontol UNESP. 2019;48:e20180128. https://dx.doi.org/10.1590/18072577.12818
\end{abstract}

\begin{abstract}
Resumo
Introdução: A solubilidade e sorção dos materiais restauradores são considerados fatores críticos, pois podem interferir na qualidade e durabilidade das restaurações. Objetivo: Avaliar a solubilidade e sorção de água de alguns materiais restauradores. Material e método: Foram confeccionados quatorze espécimes de cada um dos seguintes materiais: Equia ${ }^{\circledR}$ Forte, Z100, Fuji IX/ e Vidrion R, os quais foram levados a uma dessecadora e pesados diariamente até estabilização. Em seguida, foram inseridos em recipientes individuais contendo $40 \mathrm{~mL}$ de água destilada cada e divididos em dois grupos de acordo com o tempo de armazenagem: 7 ou 30 dias. Ao final de cada período, os espécimes foram retirados da água, pesados, levados à dessecadora e pesados novamente até estabilização. Os resultados foram analisados estatisticamente utilizando os testes ANOVA 2 critérios e Tukey. Resultado: Com exceção do Equia ${ }^{\circledR}$ Forte e Z100, os demais materiais, apresentaram uma variação significativa da solubilidade ao longo do tempo. Quanto à sorção, observa-se que apenas o material Z100 não apresentou diferença significativa em relação ao tempo de armazenagem, mas, na comparação entre os materiais, todos apresentaram diferenças significativas em ambos os períodos. Conclusão: Após os períodos de armazenagem de 7 e 30 dias em água, os materiais restauradores Equia ${ }^{\circledR}$ Forte e Z100 não apresentam variação significativa da solubilidade, sendo mais estáveis que os materiais Fuji IX e Vidrion R. Os materiais à base de ionômero de vidro, Equia ${ }^{\circledR}$ Forte, Fuji IX e Vidrion R, sofrem mais sorção em água quando comparados ao material Z100 em ambos os períodos.
\end{abstract}

Descritores: Solubilidade; cimentos de ionômeros de vidro; resinas compostas; tratamento dentário restaurador sem trauma.

\begin{abstract}
Introduction: The solubility and sorption parameters of restorative materials are considered critical factors since they may interfere with the quality and durability of restorations. Objective: The aim of this study was to evaluate the solubility and water sorption of some restorative materials. Material and method: Fourteen specimens were made $(10.0 \mathrm{~mm}$ x $2.0 \mathrm{~mm})$ of each of the following material: Equia ${ }^{\circledR}$ Forte, Z100, Fuji IX/ e Vidrion R, then taken to a desiccator and weighed daily until stabilized. They were then placed in individual containers with $40 \mathrm{~mL}$ of distilled water each and divided into two groups according to the storage time: 7 or 30 days. At the end of each period, the specimens were removed from the water, weighed, taken to the desiccator and weighed again until stabilized. The results were analyzed statistically with two-way ANOVA and Tukey. Result: Except for Equia ${ }^{\circledR}$ Forte and Z100, the other materials presented a significant variation of solubility over time. As for sorption, it was noticed that only Z100 presented no significant difference in relation to the storage time, but in comparison of the materials, all had significant differences in both 7 and 30 days periods. Conclusion: After storage periods of 7 and 30 days in water, the restorative materials Equia ${ }^{\circledR}$ Forte and Z100 do not present significant variation of solubility, being more stable than the materials Fuji IX and Vidrion R. The glass ionomer-based materials, Equia ${ }^{\circledR}$ Forte, Fuji IX and Vidrion R, suffer more sorption in water when compared to Z100 material in both periods.
\end{abstract}

Descriptors: Solubility; glass ionomer cements; composite resins; dental atraumatic restorative treatment. 


\section{INTRODUÇÃO}

A cárie dentária ainda é um problema de saúde pública. Nas últimas décadas, o governo e as instituições não governamentais têm investido para mudar esse quadro mundial ${ }^{1} \mathrm{e}$, felizmente, alguns países desenvolvidos têm apresentado um declínio significativo, muito embora nos países menos desenvolvidos, isto também ocorra, mesmo que de forma menos evidente. No Brasil, o sistema de saúde vigente tem propiciado melhorias mesmo diante da diversidade socioeconômica da população, o que dificulta a igualdade de atendimento ${ }^{2}$. Uma melhora nos índices de CPOD foi relatada para crianças e adolescentes ${ }^{3}$, enquanto que, para adultos e idosos, os índices epidemiológicos permanecem mais altos do que o recomendado pela $\mathrm{OMS}^{4}$.

Particularmente em Saúde Coletiva, os materiais ionoméricos são muito utilizados em técnicas restauradoras, como o tratamento restaurador atraumático (ART), por suas características ${ }^{5}$ de biocompatibilidade, liberação de flúor, fácil manuseio, adesão química ao tecido dentário e baixa toxicidade. A principal vantagem do ART é permitir que pessoas que vivem em áreas de difícil acesso ou que estejam em condições sistêmicas mais fragilizadas possam se beneficiar do tratamento. No entanto, os cimentos de ionômero de vidro em geral apresentam algumas desvantagens clínicas ${ }^{6}$ que podem causar uma diminuição no tempo de vida da restauração, tais como fragilidade e sensibilidade inicial à umidade ${ }^{7}$. Os efeitos de sorção (SR) e solubilidade (SL), muito observados nos cimentos ionoméricos convencionais, podem acarretar, como prejuízo à restauração, fratura ${ }^{8}$ ou infiltração marginal ${ }^{9}$ com recidiva de cárie $^{5}$, além de perda de propriedades e translucidez ${ }^{10}$.

Recentemente foi lançado no mercado um novo sistema restaurador híbrido, o Equia Forte ${ }^{\circledR}$ (GC America inc. IL, USA). 0 sistema restaurador é composto de 3 produtos: o ionômero de vidro com adição de partículas de vidro altamente reativas, o ácido poliacrílico de alto peso molecular e um protetor resinoso. Segundo seu fabricante, o protetor resinoso possui a incorporação de um novo monômero multifuncional que produz uma matriz resinosa mais resistente e, utilizando corretamente o conjunto, o sistema restaurador possui ótimas propriedades físicas e estéticas. Por conta dessas modificações, este produto também é indicado para restaurações de classe II. Por tratar-se de um produto novo no mercado, há poucas pesquisas sobre ele e, até a presente data, nenhuma pesquisa sobre a sorção de líquidos e solubilidade desse material.

Os efeitos de SR e SL também podem afetar as restaurações em resina composta, reduzindo suas propriedades e afetando seu desempenho clínico ${ }^{11}$.

Diversos estudos foram realizados utilizando compósitos e materiais ionoméricos para avaliar esses efeitos, porém a literatura não apresenta muitos dados que se correlacionam ao presente trabalho devido às diferenças de tempo e soluções utilizadas, por exemplo ${ }^{12}$. Ainda assim, sabese que é possível inferir que a SR e SL dos materiais resinosos são dependentes do tipo de monômeros e polímeros que os compõem ${ }^{13}$.

Tanto os cimentos de ionômero de vidro quanto os cimentos de ionômero de vidro modificados por resina possuem a mesma ordem de magnitude em relação aos coeficientes de difusão, quando são analisadas absorção e perda de água ${ }^{14}$. Um problema que o cimento de ionômero de vidro convencional apresenta e que pode justificar esse comportamento é a desintegração da camada superficial após a absorção de água. Já a incorporação de resina ao material diminui a difusão da água no cimento ${ }^{11}$.

Neste contexto, o objetivo deste trabalho foi avaliar a solubilidade e sorção em água de alguns materiais restauradores diretos. 


\section{MATERIAL E MÉTODO}

\section{Delineamento Experimental}

Avaliou-se a SR e SL de quatro materiais restauradores, cujas especificações são apresentadas na Tabela 1 , após períodos de 7 e 30 dias de armazenagem em água destilada, a $37^{\circ} \pm 2 \stackrel{\circ}{ } \mathrm{C}$. Para tanto, foram confeccionados sete $(n=7)$ espécimes de cada material para cada período de tempo avaliado.

Tabela 1. Composição dos materiais testados

\begin{tabular}{|c|c|c|c|c|}
\hline $\begin{array}{c}\text { Material } \\
\text { (Fabricante) }\end{array}$ & Abreviatura & Classificação & Composição & $\begin{array}{c}\text { Número do } \\
\text { Lote }\end{array}$ \\
\hline $\begin{array}{l}\text { Z100 (3M } \\
\text { ESPE. } \\
\text { Minnesota, } \\
\text { USA) }\end{array}$ & $\mathrm{Z} 100$ & $\begin{array}{c}\text { Resina } \\
\text { composta } \\
\text { microhíbrida }\end{array}$ & $\begin{array}{l}\text { Bisfenol-A-glicidilmetacrilato (BisGMA), } \\
\text { trietilenoglicoldimetacrilato(TEGDMA), } \\
\text { zircônia/sílica }\end{array}$ & 1631300188 \\
\hline $\begin{array}{l}\text { Vidrion R (SS } \\
\text { White. Rio de } \\
\text { Janeiro, BR) }\end{array}$ & VIDR & $\begin{array}{l}\text { Cimento de } \\
\text { ionômero de } \\
\text { vidro } \\
\text { convencional }\end{array}$ & $\begin{array}{l}\text { Pó: fluorsilicato de sódio cálcio alumínio, } \\
\text { sulfato de bário, ácido poliacrílico, pigmentos. } \\
\text { Líquido: ácido tartárico, água destilada }\end{array}$ & 0190716 \\
\hline $\begin{array}{l}\text { Equia }{ }^{\circledR} \text { Forte } \\
\text { (GC America } \\
\text { inc. Illinois, } \\
\text { USA) }\end{array}$ & EQUI & $\begin{array}{l}\text { Cimento de } \\
\text { ionômero de } \\
\text { vidro de alta } \\
\text { viscosidade }\end{array}$ & $\begin{array}{c}\text { Pó: Fluoro-alumino-silicato de vidro, ácido } \\
\text { poliacrílico em pó, pigmento. Líquido: ácido } \\
\text { poliacrílico, água destilada, ácido carboxílico } \\
\text { polibásico }\end{array}$ & 160120AS \\
\hline $\begin{array}{l}\text { FUJI IX (GC } \\
\text { Corporation. } \\
\text { Tokyo, JP) }\end{array}$ & FUJI & $\begin{array}{l}\text { Cimento de } \\
\text { ionômero de } \\
\text { vidro de alta } \\
\text { viscosidade }\end{array}$ & $\begin{array}{l}\text { Pó: vidro de silicato de flúor-alumínio; pó de } \\
\text { ácido poliacrílico. Líquido: ácido poliacrílico; } \\
\text { água }\end{array}$ & 1605031 \\
\hline
\end{tabular}

\section{Confecção dos Espécimes}

Para a confecção das amostras, utilizou-se uma matriz de aço com um orifício central contendo um anel justaposto com 10,0 $\mathrm{mm}$ de diâmetro e 2,0 $\mathrm{mm}$ de espessura. Para a manipulação dos materiais, seguiram-se as recomendações dos fabricantes. A inserção do material nas matrizes foi realizada com o auxílio de uma espátula de inserção, no caso da resina Z100, e com um aplicador de pontas descartáveis Precision (Maquira, Paraná, BR) para os espécimes VIDR e FUJI. O EQUI vem em cápsulas prontas para inserção. Uma tira de papel celofane foi colocada sobre uma placa de vidro e a matriz foi posicionada para a inserção do material. Em seguida foi pressionada levemente uma tira de matriz de celofane para remover os excessos do material recém-inserido na matriz. Para os espécimes de Z100, procedeu-se à fotoativação durante 40 segundos com um aparelho VALO ${ }^{\circledR}$ Cordless (Ultradent, Utah, USA). Para o EQUI, VIDR e FUJI, esperou-se o tempo de endurecimento inicial recomendado pelo fabricante. Na sequência, as amostras foram retiradas da matriz e os excessos foram removidos com uma lâmina de bisturi.

\section{Armazenagem dos Espécimes}

Após sua confecção, os espécimes foram colocados em frascos individuais, sem tampa e devidamente identificados, e esses frascos foram transferidos para um dessecador (Pyrex, São Paulo, Brasil) contendo sílica gel, a $37^{\circ} \pm 2 \stackrel{\circ}{ } \mathrm{C}$, durante 22 horas. Após este período, os espécimes foram transferidos para outro dessecador contendo sílica gel a uma temperatura de $23^{\circ} \pm 1 \stackrel{\circ}{\circ}$ por 2 horas. Os espécimes foram pesados em balança analítica GR202 (A\&D, Tokyo, JP), calibrada para $0,0001 \mathrm{~g}$ e colocados novamente na dessecadora, repetindo-se o ciclo a cada 24 horas, até se 
obter uma massa constante (m1), ou seja, até que a perda de massa total de cada espécime não fosse superior a $0,1 \mathrm{mg}$ entre cada período.

\section{Avaliação da SL e SR}

Após a secagem, os espécimes foram medidos, sendo duas medidas do diâmetro, realizadas em lados opostos, e cinco medidas da espessura, realizadas em diferentes pontos. Para isso, utilizou-se um paquímetro digital (Mitutoyo, Kanagawa, JP). Calcularam-se, então, a média do diâmetro e a média da espessura e, com esses dados, a área de superfície de cada espécime, em milímetros quadrados, e o volume (V), em milímetros cúbicos.

Após a obtenção dos valores de $\mathrm{m} 1$ e $\mathrm{V}$, os espécimes foram colocados novamente em seus respectivos frascos identificados e imersos em $40 \mathrm{ml}$ de água destilada, tomando-se o cuidado para que os espécimes tivessem a maior área possível em contato com a água. Os frascos foram fechados e armazenados em uma estufa a $37^{\circ} \pm 2{ }^{\circ} \mathrm{C}$ durante 7 ou 30 dias de acordo com cada grupo. Após este período, os espécimes foram removidos da estufa e de seus recipientes, lavados em água corrente, levemente secos com papel absorvente, expostos ao ar por 1 minuto e pesados na sequência, obtendo-se o valor de massa $\mathrm{m} 2$. Em seguida, os espécimes foram novamente acondicionados em seus frascos e colocados no dessecador onde permaneceram por 24 horas, repetindo-se o ciclo de dessecação, como feito anteriormente, até obter uma massa constante (m3).

Os valores de SR e SL foram calculados em microgramas por milímetro cúbico $\left(\mu \mathrm{g} / \mathrm{mm}^{3}\right)$, utilizando as seguintes Equações $(1,2)$ :

$\mathrm{W} \mathrm{SL}=\mathrm{ml}-\mathrm{m} 3 / \mathrm{V}$

Onde: W SL corresponde à solubilidade $\left(\boldsymbol{\mu g} / \mathrm{mm}^{3}\right) ; \mathrm{m} 1$ à massa inicial, em microgramas, antes da imersão em água; m3 à massa, em microgramas, após a segunda dessecação e V, ao volume da amostra, em milímetros cúbicos.

$\mathrm{W} S \mathrm{R}=\mathrm{m} 2-\mathrm{m} 3 / \mathrm{V}$

Onde: W SR corresponde à SR $\left(\boldsymbol{\mu} \mathrm{g} / \mathrm{mm}^{3}\right) ; \mathrm{m} 2$ à massa, em microgramas, dos espécimes após imersão em água; $\mathrm{m} 3$ à massa, em microgramas, após a segunda dessecação e $\mathrm{V}$, ao volume da amostra, em milímetros cúbicos.

Após o cálculo final dos valores de SL e SR, os dados foram analisados estatisticamente utilizando o programa R. Foi realizado o teste de Shapiro Wilk para determinar se os dados obtidos seguem o padrão de normalidade e, em seguida, realizado o teste ANOVA 2 critérios tanto para SR como para SL e, como post-hoc, foi utilizado o teste de Tukey com nível de significância de 0,05 .

\section{RESULTADO}

Os valores encontrados de SL e SR são apresentados na Tabela 2.

Tabela 2. Média (desvio padrão) da SL e SR inicial e final, após armazenagem em água ( $N=7$ ). Valores em $\mu \mathrm{g} / \mathrm{mm}^{3}$

\begin{tabular}{|c|c|c|c|c|}
\hline \multirow{2}{*}{ MATERIAL } & \multicolumn{2}{|c|}{ SOLUBILIDADE } & \multicolumn{2}{|c|}{ SORÇÃO } \\
\hline & 7 DIAS & 30 DIAS & 7 DIAS & 30 DIAS \\
\hline $\mathrm{Z} 100$ & $-7,49(0,82) \mathrm{Aa}$ & $-6,62(0,48) \mathrm{Aa}$ & $6,42(1,87) \mathrm{Aa}$ & $14,25(0,54) \mathrm{Aa}$ \\
\hline EQUI & $-29,50(6,59) \mathrm{Ba}$ & $-30,33(5,31) \mathrm{Ba}$ & $97,75(5,97) \mathrm{Ba}$ & $112,81(15,42) \mathrm{Bb}$ \\
\hline FUJI & $24,17(2,86) \mathrm{Ca}$ & $-32,27(5,84) \mathrm{Bb}$ & $126,11(10,35) \mathrm{Ca}$ & $89,73(18,33) \mathrm{Cb}$ \\
\hline VIDR & $46,67(3,71) \mathrm{Da}$ & $-23,26(2,44) \mathrm{Cb}$ & $170,50(3,39) \mathrm{Da}$ & $152,22(11,57) \mathrm{Db}$ \\
\hline
\end{tabular}

Médias com a mesma letra, maiúsculas para comparações entre as linhas (materiais) e minúsculas para comparações entre as colunas (tempo) não apresentam diferenças estatisticamente significantes $(p>0,05) . N=7$ espécimes/grupo.

Em relação à SL, observou-se que, em relação aos 7 e 30 dias, o EQUI e Z100 foram os únicos materiais que não apresentaram diferenças significativas $(p>0,05)$. Comparando os diferentes 
materiais, no período de 7 dias, houve diferença significativa entre todos os materiais $(p<0,05)$. No período de 30 dias, não houve diferença significativa entre os materiais EQUI e FUJI $(p>0,05)$.

Quanto à SR, observa-se que apenas o material Z100 não apresentou diferença significativa em relação ao tempo de armazenagem $(p>0,05)$. Já, tratando-se da comparação entre os materiais, todos tiveram diferenças significativas tanto no período de 7 dias quanto no período de 30 dias $(p<0,05)$.

\section{DISCUSSÃO}

O material EQUI não sofreu SL em nenhum dos tempos testados e mostrou-se superior neste quesito em relação aos outros materiais ionoméricos. Quanto à SR, o material apresenta valores do mesmo grau de magnitude dos outros materiais ionoméricos.

Um dos fatores que influencia a SR de líquidos é o processo de manipulação manual dos cimentos de ionômero de vidro que pode levar à formação de porosidades, o que consequentemente aumenta o processo de SR de líquido pelo materialli5,16.

Os resultados de SL e SR do material Z100 estão de acordo com a ISO 4049200917 que define que os valores de SR e SL para um material resinoso de base polimérica devem ser menores ou iguais a $40 \mu \mathrm{g} / \mathrm{mm}^{3}$ e $7,5 \mu \mathrm{g} / \mathrm{mm}^{3}$, respectivamente. Estes resultados reafirmam a escolha do material como grupo controle.

É difícil comparar os resultados de SL e SR obtidos neste estudo com os resultados de outros estudos $5,8,10-12,16,18,19$, sobre SR e SL, pois a metodologia empregada nem sempre é a mesma12; ainda, há diferenças nos períodos de tempo analisados e tamanho dos espécimes confeccionados o que resulta em diferenças no período de tempo necessário para que a água infiltre completamente a matriz polimérica12. Mesmo que a ISO 4049200917 dite os padrões de tamanho e passos a seguir ao confeccionar os espécimes e realizar os testes laboratoriais, diversos estudos ainda utilizam corpos de prova com diâmetro e espessura diferentes do que o proposto e, inclusive, realizam testes de SR e SL de maneira diferente, como é o caso dos estudos que não utilizam a fase de dessecação antes do período de imersão do espécime no líquido selecionado para o estudo.

O estudo de Mustafa et al. ${ }^{18}$ concluiu que realizar a dessecação antes do período de imersão teve efeito significativo na SR, SL e expansão volumétrica dos materiais ionoméricos testados. Isso ocorre, pois, realizar a dessecação inicial para fins de mensurar a massa seca inicial do espécime pode resultar em defeitos estruturais que afetam a SR e a SL dos materiais ionoméricos ${ }^{19}$. Ainda assim, no presente trabalho, a dessecação inicial foi realizada para seguir corretamente os padrões ditados pela ISO 4049200917.

O tempo de armazenagem utilizado no presente trabalho foi suficiente para observar a SR e SL dos materiais. Segundo Chutinan et al. ${ }^{20}$, a SR da água ocorre nas duas primeiras semanas de forma mais intensa. 0 material à base de cimento de ionômero de vidro apresenta caráter hidrofílico e sofre frequente SR de água. Dessa forma, clinicamente, é necessário que a restauração seja imediatamente protegida quando finalizada e essa proteção pode ser feita lançando mão de materiais como vaselina sólida, vernizes ou adesivos de baixa viscosidade ${ }^{21}$.

O conjunto EQUI é composto pelo material restaurador ionomérico e por um coat (protetor) resinoso. 0 protetor resinoso é responsável por realizar a proteção indicada para a restauração ionomérica, sendo uma de suas funções a de criar uma barreira entre o material ionomérico e a saliva. Segundo a ISO 4049200917 , utilizar qualquer material protetor interfere na obtenção dos verdadeiros valores de SR e SL do material ionomérico. Portanto, no presente trabalho, não se realizou a cobertura do espécime pelo protetor resinoso para que pudéssemos, de fato, testar o material ionomérico sem interferências. 
A água tem um importante papel nos cimentos de ionômero de vidro. Ela contribui para o transporte de cátions de cálcio e alumínio, reagindo com o ácido poliacrílico para formar a matriz de poliacrilato. Durante o estágio inicial de maturação, a contaminação por umidade leva à perda de componentes, diminuição das propriedades físicas e perda de translucidez. Após o tempo de presa, a dessecação e a consequente perda de água levam à inadequação das reações e à fissuração da superfície. Portanto, espera-se que os cimentos de ionômero de vidro absorvam e percam água mais facilmente que materiais resinosos ${ }^{22}$, como mostram os resultados de SR dos materiais EQUI, VIDR e FUJI se comparados ao material Z100.

A absorção e perda de água nos materiais restauradores ionoméricos se dão por um processo de difusão que obedece à lei de Fick nas primeiras $4-5$ horas. Os coeficientes de difusão dos cimentos de ionômero de vidro possuem a mesma ordem de magnitude dos cimentos de ionômero de vidro modificados por resina ${ }^{14}$. Para estes materiais, o equilíbrio de absorção de água pode estar relacionado ao potencial químico da água no meio circundante. 0 potencial químico da água pura é maior do que em soluções de cloreto de sódio, por exemplo, portanto a força motriz termodinâmica para SR de água é maior em água pura do que em soluções de sais ${ }^{23}$.

Duas abordagens para esses problemas são amplamente mencionadas na literatura. A primeira é a teoria volumétrica livre que propõe que o equilíbrio da concentração de água é regido principalmente pela fração de volume disponível em que a água se difunde através de microporosidades ou outros defeitos morfológicos sem qualquer conexão com regiões polares de moléculas presentes no material. Já a teoria da interação diz que as moléculas de água seriam ligadas sucessivamente a grupos polares de cadeias poliméricas, especialmente aquelas que formam ligações de hidrogênio como as hidroxilas ${ }^{24}$.

Assumiu-se, então, que as duas abordagens poderiam ser válidas, sendo cada uma para uma família de amostras restritas ou ocorrendo simultaneamente ${ }^{24}$. Porém, os polímeros absorvem água em diferentes quantidades, dependendo dos seus aspectos moleculares e microestruturais como a polaridade da estrutura molecular, presença de hidroxilas capazes de formar ligações de hidrogênio com a água, presença de água atraindo outras moléculas de água, dimensão, volume e difusividade das partículas de carga ${ }^{25}$. Portanto ocorrem dois mecanismos distintos: o primeiro é a SR de água, o que faz com que haja um aumento de peso do material. 0 segundo é a dissolução de materiais na água, o que leva a uma redução de peso em relação ao peso inicial após a dessecação ${ }^{11}$.

A composição do material desempenha um papel importante em suas propriedades físicoquímicas. Considerando a SR e a SL, as diferenças na composição dos cimentos de ionômero de vidro determinam o comportamento dos materiais. A presença de porosidades, o grau de conversão, o sistema iniciador da polimerização, seja ele químico ou físico, e a densidade da cadeia polimérica formada nesses materiais são fatores que desempenham um papel importante nos processos de SR e SL ${ }^{5}$. Os valores de SR e SL também dependem do tipo e conteúdo de carga, da concentração das partículas de carga, do tamanho médio das partículas dos agentes de acoplamento, da natureza das partículas de carga e do tipo de solvente ${ }^{12}$.

O material EQUI possui patente ativa e, portanto, não tem toda sua composição divulgada. 0 que se sabe até então sobre a composição desse material é que há outros componentes além dos que foram previamente citados na Tabela 1. Isso faz com que ainda não seja possível elucidar o que influencia seus mecanismos de SR e SL, limitando a discussão. Com isso, sugere-se que sejam realizados novos estudos para elucidar esses mecanismos após a divulgação da composição completa do material.

Uma limitação em relação à metodologia deste trabalho é que a medida de diferença das massas não representa exatamente a SL do material. Essa diferença também registra os processos de desintegração, como a perda de partículas dos materiais na água durante sua armazenagem. Portanto, o resultado final é uma combinação desses dois processos e não há uma diferenciação clara entre eles ${ }^{18}$. 
Outra limitação relacionada a este estudo é que não foram realizados outros testes para avaliar melhor o comportamento do EQUI. Tem-se este fato como limitação pois sabe-se que apenas a SR e SL de um material não determinam seu comportamento clínico.

\section{CONCLUSÃO}

Conclui-se que após os períodos de armazenagem de 7 e 30 dias em água os materiais restauradores Equia ${ }^{\circledR}$ Forte e Z100 não apresentam variação significativa da solubilidade, sendo mais estáveis que os materiais Fuji IX e Vidrion R. Os materiais à base de ionômero de vidro, Equia ${ }^{\circledR}$ Forte, Fuji IX e Vidrion R, sofrem mais sorção em água quando comparados ao material Z100 em ambos os períodos.

\section{REFERÊNCIAS}

1. Kassebaum NJ, Smith AGC, Bernabé E, Fleming TD, Reynolds AE, Vos T, et al. Global, regional, and national prevalence, incidence, and disability-adjusted life years for oral conditions for 195 countries, 1990 - 2015: a systematic analysis for the global burden of diseases, injuries, and risk factors. J Dent Res. 2017 Apr;96(4):380-7. http://dx.doi.org/10.1177/0022034517693566. PMid:28792274.

2. Pucca GA Jr, Gabriel M, Araujo ME, Almeida FC. Ten years of a national oral health policy in Brazil: innovation, boldness, and numerous challenges. J Dent Res. 2015 Oct;94(10):1333-7. http://dx.doi.org/10.1177/0022034515599979. PMid:26316461.

3. Roncalli AG, Sheiham A, Tsakos G, Watt RG. Socially unequal improvements in dental caries levels in Brazilian adolescents between 2003 and 2010. Community Dent Oral Epidemiol. 2015 Aug;43(4):31724. http://dx.doi.org/10.1111/cdoe.12156. PMid:25660728.

4. Pinto RS, Matos DL, Loyola AI Fo. Características associadas ao uso de serviços odontológicos públicos pela população adulta brasileira. Cien Saude Colet. 2012 Fev;17(2):531-44. http://dx.doi.org/10.1590/S1413-81232012000200026. PMid:22267047.

5. Lima RBW, Farias JFG, Andrade AKM, Silva FDSCM, Duarte RM. Water sorption and solubility of glass ionomer cements indicated for atraumatic restorative treatment considering the time and the $\mathrm{pH}$ of the storage solution. RGO Rev Gaúch Odontol. 2018 Jan;66(1):29-34. http://dx.doi.org/10.1590/1981863720180001000043100 .

6. Prabhakar AR, Sekhar VR, Kurthukoti AJ. Leaching of ions from materials used in alternative restorative technique under neutral and acidic conditions: a comparative evaluation. J Clin Pediatr Dent. 2009;34(2):125-30. http://dx.doi.org/10.17796/jcpd.34.2.y0860544254x7371. PMid:20297702.

7. Hattab FN, Amin WM. Fluoride release from glass ionomer restorative materials and the effects of surface coating. Biomaterials. 2001 Jun;22(12):1449-58. http://dx.doi.org/10.1016/S01429612(00)00253-2. PMid:11374443.

8. Bhatia HP, Singh S, Sood S, Sharma N. A comparative evaluation of sorption, solubility, and compressive strength of three different glass ionomer cements in artificial saliva: an in vitro study. Int J Clin Pediatr Dent. 2017 Jan-Mar;10(1):49-54. http://dx.doi.org/10.5005/jp-journals-10005-1407. PMid:28377656.

9. Saghiri MA, Asgar K, Lotfi M, Karamifar K, Neelakantan P, Ricci JL. Application of mercury intrusion porosimetry for studying the porosity of mineral trioxide aggregate at two different $\mathrm{pH}$. Acta Odontol Scand. 2012 Jan;70(1):78-82. http://dx.doi.org/10.3109/00016357.2011.597777. PMid:21728747.

10. Cefaly DFG, Wang L, Mello LLCP, Santos JL, Santos JR, Lauris JRP. Water sorption of resin-modified glass-ionomer cements photoactivated with LED. Braz Oral Res. 2006 Oct-Dec;20(4):342-6. http://dx.doi.org/10.1590/S1806-83242006000400011. PMid:17242796. 
11. Toledano M, Osorio R, Osorio E, Fuentes V, Prati C, García-Godoy F. Sorption and solubility of resinbased restorative dental materials. J Dent. 2003 Jan;31(1):43-50. http://dx.doi.org/10.1016/S03005712(02)00083-0. PMid:12615019.

12. Meşe A, Burrow MF, Tyas MJ. Sorption and solubility of luting cements in different solutions. Dent Mater J. 2008 Sep;27(5):702-9. http://dx.doi.org/10.4012/dmj.27.702. PMid:18972787.

13. Alshali RZ, Salim NA, Satterthwaite JD, Silikas N. Long-term sorption and solubility of bulk-fill and conventional resin-composites in water and artificial saliva. J Dent. 2015 Dec;43(12):1511-8. http://dx.doi.org/10.1016/j.jdent.2015.10.001. PMid:26455541.

14. Nicholson JW, Czarnecka B. Kinetic studies of water uptake and loss in glass-ionomer cements. J Mater Sci Mater Med. 2008 Apr;19(4):1723-7. http://dx.doi.org/10.1007/s10856-007-3244-x. PMid:17768671.

15. Malkoç MA, Sevimay M, Tatar I, Çelik HH. Micro-CT Detection and characterization of porosity in luting cements. J Prosthodont. 2015 Oct;24(7):553-61. http://dx.doi.org/10.1111/jopr.12251. PMid:25557068.

16. Gavranović-Glamoč A, Ajanović M, Korać S, Zukić S, Strujić-Porović S, Kamber-Ćesir A, et al. Evaluation of the water sorption of luting cements in different solutions. Acta Med Acad. 2017 Nov;46(2):124-32. PMid:29338276.

17. International Organization for Standardization, Technical Committee. ISO/TC 106/SC 1. Dentistrypolymer-based restorative materials (ISO 4049). 4th ed. Geneva: ISO; 2009.

18. Mustafa R, Alshali RZ, Silikas N. The effect of desiccation on water sorption, solubility and hygroscopic volumetric expansion of dentine replacement materials. Dent Mater. 2018 Aug;34(8):e205-13. http://dx.doi.org/10.1016/j.dental.2018.05.012. PMid:29805062.

19. Pieper CM, Zanchi CH, Rodrigues-Junior SA, Moraes RR, Pontes LS, Bueno M. Sealing ability, water sorption, solubility and toothbrushing abrasion resistance of temporary filling materials. Int Endod J. 2009 Oct;42(10):893-9. http://dx.doi.org/10.1111/j.1365-2591.2009.01590.x. PMid:19549149.

20. Chutinan S, Platt JA, Cochran MA, Moore BK. Volumetric dimensional change of six direct core materials. Dent Mater. 2004 May;20(4):345-51. http://dx.doi.org/10.1016/S0109-5641(03)00127-1. PMid:15019448.

21. Sidhu SK, Nicholson JW. A review of glass-ionomer cements for clinical dentistry. J Funct Biomater. 2016 Jun;7(3):16. http://dx.doi.org/10.3390/jfb7030016. PMid:27367737.

22. Tavangar MS, Jafarpur D, Bagheri R. Evaluation of compressive strength and sorption/solubility of four luting cements. J Dent Biomater. 2017 Jun;4(2):387-93. PMid:28959770.

23. Nicholson JW. The physics of water sorption by resin-modified glass-ionomer dental cements. J Mater Sci Mater Med. 1997 Nov;8(11):691-5. http://dx.doi.org/10.1023/A:1018587907243. PMid:15348820.

24. Mortier E, Gerdolle DA, Dahoun A, Panighi MM. Influence of initial water content on the subsequent water sorption and solubility behavior in restorative polymers. Am J Dent. 2005 Jun;18(3):177-81. PMid:16158809.

25. Marcovich NE, Reboredo MM, Aranguren MI. Moisture diffusion in polyester-woodflour composites. Polymer (Guildf). 1999 Dec;40(26):7313-20. http://dx.doi.org/10.1016/S0032-3861(99)00093-2.

\section{CONFLITOS DE INTERESSE}

Os autores declaram não haver conflitos de interesse. 


\section{*AUTOR PARA CORRESPONDÊNCIA}

Raquel Sano Suga Terada, UEM - Universidade Estadual de Maringá, Departamento de Odontologia, Avenida Mandacaru, 1550, 87083-170 Maringá - PR, Brasil, e-mail: rssterada@uem.br

Recebido: Novembro 23, 2018

Aprovado: Abril 24, 2019 